EXTENDED REPORT

\title{
The relation between $C$ reactive protein and age related macular degeneration in the Cardiovascular Health Study
}

\author{
G McGwin, T A Hall, A Xie, C Owsley
}

Br J Ophthalmol 2005;89:1166-1170. doi: 10.1136/bjo.2005.067397

See end of article for authors' affiliations .....................

Correspondence to: Dr G McGwin, Jr, Department of Ophthalmology, School of Medicine, University of Alabama at Birmingham, $700 \mathrm{~S} 18$ th Street, Suite 609, Birmingham, AL 35294-0009, USA: mcgwin@uab.edu

Accepted for publication 23 April 2005 Aim: To test the hypothesis that individuals with age related macular degeneration (AMD) have increased
$C$ reactive protein (CRP) levels. Methods: A cross sectional study design using data from the Cardiovascular Health Study (CHS), a longitudinal study that enrolled older adults from four communities in the United States from 1989 to 1990 , was employed to investigate the existence of an association between AMD and CRP levels in this population. Fundus photographs from 1997 and 1998 were used to identify individuals with $(n=390)$ and without AMD $(n=2365)$. The association between AMD and CRP levels (measured at baseline) was compared, adjusting for the potentially confounding effect of demographic, lifestyle, and health related characteristics.

Results: Among the 2755 CHS participants with gradable fundus photographs, 390 were identified as having AMD. Overall, median CRP levels among those with AMD (1.76 mg/l) were similar to those without AMD (1.77 mg/l). CRP levels were categorised into quartiles and compared between those with and without AMD. Relative to those in the lowest quartile $(0.07-0.93 \mathrm{mg} / \mathrm{l})$, the odds ratios (OR) in the higher quartiles, adjusted for demographic, lifestyle, and health related characteristics were increased but not statistically significant $(0.94-1.77 \mathrm{mg} / \mathrm{l}: \mathrm{OR}=1.14,95 \% \mathrm{Cl} 0.82$ to $1.60 ; 1.78-3.04 \mathrm{mg} / \mathrm{l}$ : $\mathrm{OR}=1.24,95 \% \mathrm{Cl} 0.88$ to $1.75 ;>3.04 \mathrm{mg} / \mathrm{l}: \mathrm{OR}=1.24,95 \% \mathrm{Cl} 0.87$ to 1.78 ).

Conclusions: In the CHS, there is no evidence that CRP levels are associated with AMD. These data do not support the theory alleging non-specific systemic inflammation in the aetiology and natural history of this disease.
$\mathrm{T}$ he aetiology of age related macular degeneration (AMD) is not well understood. Many theories exist and feature mechanisms of oxidative stress, atherosclerotic-like changes, genetic predisposition, and inflammation. ${ }^{1-4}$ Recently, the role of inflammation has received considerable attention. $^{2-7}$ One of the pathological hallmarks of AMD is the focal deposition of extracellular material between the retinal pigmented epithelium (RPE) and Bruch's membrane. This material, referred to as drusen, is known to be composed of several cellular and humoural constituents of systemic inflammatory and immune mediated processes (for example, HLA-DR, immunoglobulin lambda and kappa light chains, complement component 5 and 9, amyloid A, amyloid P component, fibrinogen, vitronectin, and $\mathrm{C}$ reactive protein). ${ }^{248}$ Moreover, the accumulation of drusen can damage the surrounding structures, including Bruch's membrane, and is associated with visual deficits that precede the loss of visual acuity in AMD. ${ }^{4}$ Although information continues to mount providing evidence that drusen formation and the development of AMD are mediated through inflammatory processes, how inflammation plays a role in the aetiology and natural history of AMD remains unclear.

The epidemiological research regarding the relation between inflammation and AMD has been less prolific than laboratory based investigations. Although AMD has been shown to be associated with systemic inflammatory disease (for example, gout, obesity) and its indication (for example, elevated leukocyte count, elevated fibrinogen level), ${ }^{6} 1011$ these findings have not been consistent. ${ }^{12-14}$ The use of antiinflammatory medications and their association with AMD has also produced mixed results. ${ }^{15-22}$ A significant limitation of these earlier investigations has been the use of non-specific indicators of inflammation (for example, anti-inflammatory medication use or specific inflammatory diseases). An exception is the recent investigation by Seddon et al that suggested an increased $C$ reactive protein (CRP) level was an independent risk factor for AMD. ${ }^{5}$

The objective of the current study is to further evaluate the association between CRP and AMD using data from the Cardiovascular Health Study (CHS). Although a null association between CRP and AMD was previously reported using these data, ${ }^{23}$ this conclusion was reported by simply stating no association existed without providing the actual results. Moreover, an evaluation of smoking by stratification, reported to be an important factor in a recent review article, ${ }^{22}$ was not performed. Despite the reported lack of statistical significance, the actual pattern of the results may still yield important information regarding the potential for a relationship to exist.

\section{PATIENTS AND METHODS \\ Patients}

The CHS is a population based, prospective cohort study of cardiovascular disease among adults aged 65 years and older. Details regarding the study design and subject recruitment are described elsewhere. ${ }^{24}$ Study participants were recruited in 1989 and 1990 from four locations across the United States that included Allegheny County, Pennsylvania; Forsyth County, North Carolina; Sacramento County, California; and Washington County, Maryland. Potentially eligible study subjects were identified using Medicare eligibility lists from these counties; from these lists random samples were selected. A total of 5888 subjects were recruited (5201 in

Abbreviations: $A M D$, age related macular degeneration; $B M I$, body mass index; BRB, blood retinal barrier; CHS, Cardiovascular Health Study; CRP, C reactive protein; HDL, high density lipoprotein; LDL, low density lipoprotein; RPE, retinal pigmented epithelium. 
1989 and 1990 and an additional 687 black subjects from Forsyth, Sacramento, and Allegheny Counties in 1992 and 1993). Differences between those who did and did not participate in the study have been reported elsewhere. ${ }^{25}$ At baseline and annually thereafter, physical and laboratory evaluations were performed.

\section{Subject selection}

One of the primary variables of interest for this study is AMD status. To ascertain the presence of AMD and other retinal diseases, retinal photography was added to the study protocol for the 1997-98 examination. Retinal photographs were taken of one randomly selected eye. Participants spent 5 minutes in a dark room before photographs were taken. The retinal photography protocol was similar to those reported for the Atherosclerosis Risk in Communities study that has been previously described. ${ }^{26}{ }^{27}$ Of the 4447 surviving participants, 4249 participated in the 1997-98 examination; however, only 3074 of these participants were examined at a study clinic wherein fundus photography was performed. Of these participants, retinal photographs were available on 2755; this is the segment of the cohort used in this study. A comparison between those surviving cohort members with and without fundus photographs has been previously published. $^{23}$

\section{Variable selection and definition}

The presence of AMD was defined based upon information obtained from the retinal photographs. Photographs were graded in a masked fashion using a modified Wisconsin ARM classification scheme as previously described. ${ }^{28} 29$ Early AMD was defined as the presence of soft drusen alone, RPE depigmentation alone, or a combination of soft drusen with increased retinal pigment and/or depigmentation in the absence of late AMD. Late AMD was defined as the presence of exudative macular degeneration or pure geographic atrophy. Of the 2755 study subjects, 390 were identified as having AMD (360 early; 30 late). For the purpose of this study, cases of early and late AMD were combined into a single group, as there were too few subjects with late AMD for meaningful analysis. When the analyses were conducted including and excluding the subjects with late AMD, the study results were similar.

$\mathrm{C}$ reactive protein was measured at baseline by use of an enzyme linked immunosorbent assay developed at the CHS central blood laboratory. ${ }^{30}$ It is a colorimetric competitive immunoassay that uses purified protein and polyclonal antiCRP antibodies. The interassay coefficient of variation is $5.5 \% .^{31}$

In addition to demographic characteristics (age, sex, race), information pertaining to specific behavioural and medical characteristics was also extracted from the CHS data files. With respect to behavioural characteristics, information regarding smoking (never, former, and current) and alcohol consumption (ever, never) was obtained. Variables for body mass index (BMI), the presence of selected medical conditions (diabetes, hypertension, stroke, cardiovascular disease), cholesterol levels (HDL, LDL, total), and blood pressure (systolic, diastolic) were also extracted from the CHS data files. These variables were selected as they were considered potential confounders for the association between AMD and CRP as previously described ${ }^{1012}{ }^{14}$ though not all variables previously described were available in the CHS data files. Details regarding the collection and definition of these variables are available elsewhere. ${ }^{24}$

\section{Statistical analysis}

Demographic and medical characteristics were compared between those with and without AMD using $t$ and $\chi^{2}$ tests for continuous and categorical variables, respectively. CRP was categorised into quartiles based upon the entire distribution of CRP values for the study population. With AMD status and CRP quartiles as the dependent and independent variables, respectively, logistic regression was used to calculate odds

\begin{tabular}{|c|c|c|c|}
\hline & Any AMD $(n=390)$ & No AMD $(n=2365)$ & p Value \\
\hline \multicolumn{4}{|l|}{ Demographic } \\
\hline \multicolumn{4}{|l|}{ Age (years), \% (n) } \\
\hline$<76$ & $16.4(64)$ & $28.3(668)$ & $<0.0001$ \\
\hline $76-77$ & $19.0(74)$ & $22.2(526)$ & \\
\hline $78-81$ & $28.7(112)$ & $27.2(643)$ & \\
\hline$>81$ & $35.9(140)$ & $22.3(528)$ & \\
\hline Sex, \% male (n) & $41.0(160)$ & $40.0(946)$ & 0.7019 \\
\hline Race, \% white (n) & $91.8(358)$ & 82.6 (1953) & $<0.0001$ \\
\hline \multicolumn{4}{|l|}{ Behavioural } \\
\hline Smoking status, $\%$ (n) & & & 0.8387 \\
\hline Never & 50.0 (194) & $48.4(1133)$ & \\
\hline Former & $43.3(168)$ & $44.8(1048)$ & \\
\hline Current & $6.7(26)$ & $6.9(161)$ & \\
\hline \multicolumn{3}{|l|}{ Alcohol consumption, \% (n) } & 0.3866 \\
\hline Never & $45.1(176)$ & $42.8(1011)$ & \\
\hline Ever & $54.9(214)$ & $57.2(1352)$ & \\
\hline \multicolumn{4}{|l|}{ Medical } \\
\hline BMI $\left(\mathrm{kg} / \mathrm{m}^{2}\right)$, mean $(\mathrm{SD})$ & $26.3(3.9)$ & $26.8(4.0)$ & 0.0215 \\
\hline \multicolumn{4}{|l|}{ Selected medical conditions, \% (n) } \\
\hline Diabetes & $9.9(38)$ & $13.2(309)$ & 0.0776 \\
\hline Hypertension & 48.5 (189) & $49.5(1170)$ & 0.7117 \\
\hline Stroke & $3.9(15)$ & 5.4 (127) & 0.2073 \\
\hline Cardiovascular disease & 25.9 (101) & $26.4(624)$ & 0.8395 \\
\hline HDL cholesterol (mg/dl), mean (SD) & $55.0(14.6)$ & $54.3(14.5)$ & 0.3945 \\
\hline LDL cholesterol (mg/dl), mean (SD) & $123.4(30.8)$ & $126.6(31.7)$ & 0.0679 \\
\hline Total serum cholesterol $(\mathrm{mg} / \mathrm{dll})$, mean (SD) & $200.8(31.2)$ & $204.8(33.2)$ & 0.0366 \\
\hline \multicolumn{4}{|l|}{ Blood pressure, mean (SD) } \\
\hline Systolic & $131.4(18.6)$ & $132.3(20.3)$ & 0.3645 \\
\hline Diastolic & $66.2(10.4)$ & $66.9(11.0)$ & 0.2311 \\
\hline
\end{tabular}

$A M D$, age related macular degeneration; BMI, body mass index; HDL, high density lipoprotein; LDL, low density lipoprotein; SD, standard deviation. 
Table 2 A frequency of $C$ reactive protein quartiles among $A M D$ and non-AMD subjects and associated odds ratios and $95 \%$ confidence intervals

\begin{tabular}{|c|c|c|c|c|}
\hline & \multicolumn{4}{|c|}{$C$ reactive protein groups } \\
\hline & $<0.93 \mathrm{mg} / \mathrm{l}$ & $0.93-1.77 \mathrm{mg} / \mathrm{l}$ & $1.78-3.04 \mathrm{mg} / \mathrm{l}$ & $3.05-107.97 \mathrm{mg} / \mathrm{l}$ \\
\hline $\mathrm{AMD}(\mathrm{n})$ & 97 & 98 & 99 & 95 \\
\hline Without AMD (n) & 593 & 573 & 580 & 583 \\
\hline OR $(95 \% \mathrm{Cl})^{*}$ & 1.0 & $1.05(0.77-1.42)$ & $1.04(0.77-1.41)$ & $1.00(0.73-1.35)$ \\
\hline OR $(95 \% \mathrm{Cl}) \dagger$ & 1.0 & $1.06(0.78-1.44)$ & 1.05 (0.77-1.42) & $1.09(0.80-1.49)$ \\
\hline OR $(95 \% \mathrm{Cl}) \ddagger$ & 1.0 & $1.14(0.82-1.60)$ & $1.24(0.88-1.75)$ & $1.24(0.87-1.78)$ \\
\hline
\end{tabular}

$A M D$, age related macular degeneration; n, number; $\mathrm{OR}$, odds ratio; $\mathrm{Cl}$, confidence intervals.

*Unadjusted.

†Adjusted for age, sex, and race.

$\ddagger$ Adjusted for all variables appearing in table 1 .

ratios (ORs) and associated 95\% confidence intervals (CIs). In this analysis the lowest CRP quartile was used as the referent group. ORs and 95\% CIs were calculated with and without adjustment for the potentially confounding effects of the specific demographic and medical characteristics described above. To evaluate the potential modifying effect of smoking as described by Seddon et al, ${ }^{5}$ these associations were also calculated stratified by smoking status (ever $v$ never). $p$ Values of $\leqslant 0.05$ were considered statistically significant.

\section{RESULTS}

Study participants with AMD tended to be older and were more likely to be White compared to those without AMD; there was no difference with respect to sex (table l). Smoking and alcohol consumption was also similar between the groups. Those with AMD had a significantly lower BMI though the absolute difference was very small. Hypertension and cardiovascular disease were common in both groups; diabetes and stroke less so. None of these differences was statistically significant. HDL cholesterol levels were similar among those with and without AMD. LDL and total serum cholesterol values were, on average, lower among those with AMD; however, the $\mathrm{p}$ value for LDL cholesterol was of borderline statistical significance $(p=0.0679)$. Blood pressure, both systolic and diastolic, did not differ between the groups.

Table 2 presents odds ratios (OR) and 95\% confidence intervals (CI) for the association between AMD and CRP. The unadjusted and age, sex, and race adjusted associations provide no evidence of an association between AMD and CRP. When additional adjustment for medical characteristics is performed, the ORs in each quartile increase, yet none is statistically significant. Because the CRP quartiles in the current study differed from those used by Seddon et al, ${ }^{5}$ the CRP quartile cutpoints used in their analysis were also applied to the data from the current study (table 3). The results indicate the lack of an association between AMD and CRP.

When the data are stratified according to smoking status, the unadjusted ORs suggest no significant relation between AMD and CRP for either never or ever smokers (table 4). Following adjustment for demographic and medical characteristics, for never smokers all of the ORs are increased yet the only significant association is observed for the 3rd quartile (OR 1.65; 95\% CI 1.01 to 2.69). Among ever smokers, adjustment had little impact on the magnitude of the associations.

\section{DISCUSSION}

There have been several epidemiological investigations of the relation between inflammation and AMD. Klein et al sought to examine the relation between diseases (that is, emphysema, gout) and markers (that is, white blood cell count, serum albumin, platelet count) associated with systemic inflammation and AMD. ${ }^{6}$ They reported modest associations between these diseases and markers, except platelet count, with AMD. Other similar studies have not reported consistent findings. ${ }^{12-14}$ For example, white blood cell count was found to be associated with AMD in one study ${ }^{6}$ but three others reported no such association..$^{23} 3233$ Similarly, increased plasma fibrinogen levels were associated with AMD in two studies ${ }^{1132}$ but not in two others. ${ }^{34} 35$

Our results are contrary to the only other study on this topic by Seddon et al, which reported that increased CRP levels were associated with an increased risk of AMD. ${ }^{5}$ Moreover, this study reported that advanced cases of AMD were associated with higher levels of CRP than were cases with essentially no drusen. In the Seddon et al study and the present study there was a difference in the distribution of CRP values that produced different quartiles; however, when the quartiles used by Seddon et al were applied to the CHS data, we still observed no association. Regarding cigarette smoking, Seddon et al reported that the risk of AMD was

Table 3 Frequency of $C$ reactive protein quartiles as defined by Seddon et al among $A M D$ and non-AMD subjects and associated odds ratios and $95 \%$ confidence intervals

\begin{tabular}{|c|c|c|c|c|}
\hline & \multicolumn{4}{|c|}{$\mathrm{C}$ reactive protein groups } \\
\hline & $0.2-1.2 \mathrm{mg} / \mathrm{l}$ & $1.2-2.7 \mathrm{mg} / \mathrm{l}$ & $2.7-6.5 \mathrm{mg} / \mathrm{l}$ & $6.5-117.0 \mathrm{mg} / \mathrm{l}$ \\
\hline $\mathrm{AMD}(\mathrm{n})$ & 133 & 130 & 84 & 42 \\
\hline Without AMD (n) & 783 & 844 & 452 & 250 \\
\hline OR $(95 \% \mathrm{Cl})^{*}$ & 1.0 & $0.91(0.70-1.18)$ & $1.09(0.81-1.47)$ & $0.99(0.68-1.44)$ \\
\hline OR $(95 \% \mathrm{Cl}) \dagger$ & 1.0 & $0.91(0.70-1.19)$ & $1.18(0.87-1.60)$ & $1.10(0.75-1.61)$ \\
\hline OR $(95 \% \mathrm{Cl}) \ddagger$ & 1.0 & $0.97(0.73-1.30)$ & $1.39(0.99-1.96)$ & $1.09(0.70-1.70)$ \\
\hline
\end{tabular}

$A M D$, age related macular degeneration; $n$, number; $O R$, odds ratio; $\mathrm{Cl}$, confidence intervals. *Unadjusted.

†Adjusted for age, sex, and race.

†Adjusted for all variables appearing in table 1. 
Table 4 Odds ratios and $95 \%$ confidence intervals for association between AMD and C-reactive protein according to smoking status

\begin{tabular}{|c|c|c|c|c|}
\hline & \multicolumn{4}{|c|}{$\mathrm{C}$ reactive protein groups } \\
\hline & $<0.93 \mathrm{mg} / \mathrm{l}$ & $0.93-1.77 \mathrm{mg} / \mathrm{l}$ & $1.78-3.04 \mathrm{mg} / \mathrm{l}$ & $3.05-107.97 \mathrm{mg} / \mathrm{l}$ \\
\hline \multicolumn{5}{|l|}{ Never smokers } \\
\hline OR $(95 \%$ CI)* & 1.0 & $1.09(0.71-1.69)$ & $1.32(0.86-2.02)$ & $1.17(0.75-1.81)$ \\
\hline OR $(95 \% \mathrm{Cl}) \dagger$ & 1.0 & $1.28(0.79-2.07)$ & $1.65(1.01-2.69)$ & $1.37(0.81-2.31)$ \\
\hline \multicolumn{5}{|l|}{ Ever smokers } \\
\hline OR $(95 \% \mathrm{Cl})^{*}$ & 1.0 & $1.00(0.66-1.53)$ & $0.85(0.55-1.31)$ & $0.89(0.58-1.36)$ \\
\hline OR $(95 \%$ Cl) $\dagger$ & 1.0 & $1.05(0.65-1.70)$ & $0.95(0.57-1.57)$ & $1.14(0.69-1.88)$ \\
\hline
\end{tabular}

$A M D$, age related macular degeneration; $C R P, C$ reactive protein; $O R$, odds ratio; $C l$, confidence intervals. *Unadjusted.

†Adjusted for all variables appearing in table 1.

highest among those with high CRP values who were also smokers. The prior analysis of the CHS data did not evaluate the effect of smoking on the association between CRP and AMD. ${ }^{23}$ We have redressed this issue in the current study and found, in general, that smoking had no effect on the association between CRP and AMD. It should be noted that the study by Seddon et al contained more patients with advanced AMD than in the current study and in that study CRP values were significantly increased only in this group. If CRP is only associated with advanced disease then the current study may not have had a sufficient number of subjects to detect an association.

If an association between AMD and CRP truly exists, limitations of the current study may explain why such an association was not observed. The CHS participants included in the present study represent only a subset of the entire CHS cohort. Of those alive at the 1997-98 examination, roughly $62 \%$ had a retinal photograph available and were therefore able to participate in the current study. CRP levels were higher among those who did not have a retinal photograph; ${ }^{23}$ if these individuals were also more likely to have AMD then the results of the current study may be biased towards the null. Additionally, as increased mortality has recently been reported in patients with $\mathrm{AMD},{ }^{36}$ the high mortality rate in the current study (25\%) may have reduced the number of patients with AMD and also predisposed toward a null result. It is also important to note that whereas CRP measurements were based on blood samples taken at the time of the baseline examination, retinal photographs were taken approximately 10 years later. It would have been optimal if disease status was known at the baseline examination thereby allowing for the exclusion of prevalent cases. By starting with a cohort of subjects free of AMD, the temporal relation between CRP and the incidence of AMD would have been easier to establish. On the other hand, individuals do have stable CRP concentrations over time; ${ }^{37}$ thus, although a temporal relation could not be established, the CRP levels taken during the study should represent levels before and after the retinal photographs were taken.

There is mounting evidence from laboratory based studies that inflammation plays a key role in the pathogenesis of AMD. ${ }^{1-3} 8$ In contrast, epidemiological investigations have produced equivocal results. There are several possible reasons to explain this lack of agreement between the laboratory and clinical literature, the most obvious of which is the different capabilities of laboratory and clinical settings to detect the same physiological events. The design of laboratory based studies using human donor eyes allows for markers of inflammation to be measured directly in the structures of the eye whereas clinical studies must rely on systemic measurements such as CRP and plasma fibrinogen. Moreover, given the fact that the retina makes up an extremely small part of the body and that the portion involved in AMD is even smaller, the local inflammatory reaction reported to exist therein by laboratory studies would not be expected to create detectable levels of acute phase reactants in the bloodstream anymore than a small laceration would. Therefore, if CRP or other acute phase reactants were found to be associated with $\mathrm{AMD}$, the relation would more likely be aetiological than consequential. However, the flaw in this line of reasoning is the lack of overwhelming evidence for a relation between AMD and conditions associated with an increased CRP (for example, obesity, ${ }^{38}$ hypertension, ${ }^{39-42}$ diabetes, ${ }^{43-46}$ coronary artery disease, ${ }^{37}$ and osteoarthritis ${ }^{47-49}$ ). Although the AgeRelated Eye Disease Study (AREDS) found some of these conditions (obesity, hypertension, and arthritis) to be associated with AMD, the results were modest. ${ }^{10}$ A recent study by Tomany et al pooled data from the Beaver Dam, Blue Mountains, and Rotterdam Eye studies and found that only smoking increased the risk of AMD. ${ }^{12}$ No association was found for diabetes, hypertension, obesity, or history of myocardial infarction. ${ }^{12}$ Furthermore, after a recent comprehensive examination of the existing literature concerning risk factors for AMD, Klein et al concluded that only smoking, hypertension, and cataract surgery have consistently been related to AMD. ${ }^{14}$ With respect to smoking, we did not observe an association between AMD and smoking status. However, despite the seemingly well accepted association between smoking and AMD, in reality the literature provides less consistent evidence for such an association. Although the literature provides consistent support for an association between smoking and advanced AMD and its associated lesions, there is far less support for a relation with early AMD, which were the majority of cases in the current study.

It is also important to consider the physiology of the blood retinal barrier (BRB). The BRB maintains the microenvironment of the retina through selective transport and the tight junctions of the retinal vascular endothelium and the retinal pigmented epithelium. ${ }^{3}$ This creates an immune privileged site that aggressively prevents inflammation so that the exact microanatomy required for vision can be maintained..$^{50}$ Thus, the extent to which systemic markers of inflammation could impact the localised processes in the eye, at most, would be minimal. Another significant limitation of most laboratory based research is that the observed associations are cross sectional in nature. These studies measure inflammatory markers coincident with the presence of disease. This precludes the determination of inflammation as a precursor or consequence of the disease. Of note, several observational studies also suffer from this same limitation.

In summary, the results of this study do not support an association between a systemic marker of inflammation, specifically CRP, and AMD. The inability to measure localised inflammatory changes in an intact human eye represents one of the largest obstacles to effectively investigate the mounting laboratory research suggesting a pathological role for 
inflammation in AMD. Moreover, the cross sectional nature of most research to date, both laboratory and clinical, also hampers firm conclusions from being drawn. Future observational studies should attempt to resolve this issue and evaluate the association between CRP and other markers of inflammation in a prospective manner.

\section{ACKNOWLEDGEMENTS}

This research was supported by NIH grant R21 EY14071, Research to Prevent Blindness Inc, New York, NY, USA and the EyeSight Foundation of Alabama, Birmingham, AL, USA. Cynthia Owsley is a Research to Prevent Blindness Senior Scientific Investigator.

\section{Authors' affiliations}

G McGwin, T A Hall, A Xie, C Owsley, Department of Ophthalmology, School of Medicine, University of Alabama at Birmingham, Birmingham, AL, USA

G McGwin, Department of Epidemiology and International Health, School of Public Health, University of Alabama at Birmingham, Birmingham, AL, USA

G McGwin, Section of Trauma, Burns, and Surgical Critical Care, Division of General Surgery, Department of Surgery, School of Medicine, University of Alabama at Birmingham, Birmingham, AL, USA

Competing interests: none declared

\section{REFERENCES}

1 Zarbin MA. Current concepts in the pathogenesis of age-related macular degeneration. Arch Ophthalmol 2004;122:598-614.

2 Anderson DH, Mullins RF, Hageman GS, et al. A role for local inflammation in the formation of drusen in the aging eye. Am J Ophthalmol 2002;134:411-31.

3 Penfold PL, Madigan MC, Gillies MC, et al. Immunological and aetiological aspects of macular degeneration. Prog Retin Eye Res 2001 ;203:385-414

4 Hageman G, PJ L, NH VC, et al. An integrated hypothesis that considers drusen as biomarkers of immune-mediated processes at the RPE-Bruch's membrane interface in aging and age-related macular degeneration. Prog Retin Eye Res 2001;20:705-32.

5 Seddon J, Gensler G, Milton R, et al. Association between C-reactive protein and age-related macular degeneration. JAMA 2004;291:704-10.

6 Klein R, Klein BE, Tomany SC, et al. Association of emphysema, gout, and inflammatory markers with long-term incidence of age-related maculopathy. Arch Ophthalmol 2003;121:674-78.

7 Johnson LV, Leitner WP, Staples MK, et al. Complement activation and inflammatory processes in Drusen formation and age related macular degeneration. Exp Eye Res 2001;73:887-96.

8 Johnson LV, Ozaki S, Staples MK, et al. A potential role for immune complex pathogenesis in drusen formation. Exp Eye Res 2000;70:441-9.

9 Young RW. Pathophysiology of age-related macular degeneration. Surv Ophthalmol 1987;31:291-306.

10 Group A-REDSR. Risk factors associated with age-related macular degeneration. A case-control study in the age-related eye disease study: AgeRelated Eye Disease Study Report Number 3, Ophthalmology 2000;107:2224-32

11 Smith W, Mitchell P, Leeder SR, et al. Plasma fibrinogen levels, other cardiovascular risk factors, and age-related maculopathy: the Blue Mountains Eye Study. Arch Ophthalmol 1998;116:583-7.

12 Tomany SC, Wang JJ, Van Leeuwen R, et al. Risk factors for incident agerelated macular degeneration: pooled findings from 3 continents. Ophthalmology 2004;111:1280-7.

13 Klein R, Klein BE, Tomany SC, et al. The association of cardiovascular disease with the long-term incidence of age-related maculopathy: the Beaver Dam Eye Study. Ophthalmology 2003;110:1273-80.

14 Klein R, Peto T, Bird A, et al. The epidemiology of age-related macular degeneration. Am J Ophthalmol 2004:137:486-95.

15 McCarty CA, Mukesh BN, Fu CL, et al. Risk factors for age-related maculopathy: the Visual Impairment Project. Arch Ophthalmol 2001;119:1455-62

16 McCarty CA, Mukesh BN, Guymer RH, et al. Cholesterol-lowering medications reduce the risk of age-related maculopathy progression. Med J Aust 2001:175:340.

17 McGwin G Jr. Owsley C, Curcio CA, et al. The association between statin use and age related maculopathy. Br J Ophthalmol 2003;87:1121-25.

18 Hall NF, Gale CR, Syddall H, et al. Risk of macular degeneration in users of statins: cross sectional study. BMJ 2001:323:375-6.

19 van Leeuwen R, Tomany S, Wang J, et al. Is medication use associated with the incidence of early age-related maculopathy? Pooled findings from 3 continents. Ophthalmology 2004;111:1169-75.
20 Klein R, Klein BE, Jensen SC, et al. Medication use and the 5-year incidence of early age-related maculopathy: the Beaver Dam Eye Study. Arch Ophthalmol 2001;119:1354-9.

21 Klein R, Klein BE, Tomany SC, et al. Relation of statin use to the 5-year incidence and progression of age-related maculopathy. Arch Ophthalmol 2003;121:1151-5.

22 Christen WG, Glynn RJ, Ajani UA, et al. Age-related maculopathy in a randomized trial of low-dose aspirin among US physicians. Arch Ophthalmol 2001:119:1143-9.

23 Klein R, Klein BE, Marino EK, et al. Early age-related maculopathy in the cardiovascular health study. Ophthalmology 2003;110:25-33.

24 Fried LP, Borhani NO, Enright P, et al. The Cardiovascular Health Study: design and rationale. Ann Epidemiol 1991;1:263-76.

25 Tell GS, Fried LP, Hermanson B, et al. Recruitment of adults 65 years and older as participants in the Cardiovascular Health Study. Ann Epidemiol 1993;3:358-66.

26 Hubbard LD, Brothers RJ, King WN, et al. Methods for evaluation of retina microvascular abnormalities associated with hypertension/sclerosis in the Atherosclerosis Risk in Communities Study. Ophthalmology 1999;106:2269-80

27 Group. ARIC Retainal Photography Protocol, Manual 14A, and ARIC Reading Center Protocol, Manual 14B. Chapel Hill: University of North Carolina, CB\#, 8030.

28 Klein R, Davis M, Magli YL, et al. Wisconsin age-related maculopathy grading system. Madison: University of Wisconsin Medical School, Department of Ophthalmology and Visual Sciences, 1991.

29 Klein R, Davis MD, Magli YL, et al. The Wisconsin age-related maculopathy grading system. Ophthalmology 1991;98:1128-34.

30 Cushman M, Cornell ES, Howard PR, et al. Laboratory methods and quality assurance in the Cardiovascular Health Study. Clin Chem 1995:41:264-70.

31 Macy EM, Hayes TE, Tracy RP. Variability in the measurement of C-reactive protein in healthy subjects: implications for reference intervals and epidemiological applications. Clin Chem 1997;43:52-8.

32 Klein R, Klein BE, Jensen SC, et al. Age-related maculopathy in a multiracial United States population: the National Health and Nutrition Examination Survey III. Ophthalmology 1999;106:1056-65.

33 Klein R, Clegg L, Cooper LS, et al. Prevalence of age-related maculopathy in the Atherosclerosis Risk in Communities Study. Arch Ophthalmol 1999:117:1203-10.

34 Group TEDC-CS. Risk factors for neovascular age-related macular degeneration. The Eye Disease Case-Control Study Group. Arch Ophthalmol 1992;110:1701-8.

35 Klein R. Epidemiology. In: Berger J, Fine S, Maguire M, eds. Age-related macular degeneration. St Lovis, Mo: Mosby, 1999:31-56.

36 Clemons TE, Kurinij N, Sperduto RD. Associations of mortality with ocular disorders and an intervention of high-dose antioxidants and zinc in the AgeRelated Eye Disease Study: AREDS Report No 13. Arch Ophthalmol 2004:122:716-26.

37 Pepys MB, Hirschfield GM. C-reactive protein: a critical update. J Clin Invest 2003;111:1805-12

38 Visser M, Bouter LM, McQuillan GM, et al. Elevated C-reactive protein levels in overweight and obese adults. JAMA 1999:282:2131-5.

39 Pedrinelli R, Dell'Omo G, Di Bello V, et al. Low-grade inflammation and microalbuminuria in hypertension. Arterioscler Thromb Vasc Biol 2004;24:2414-19.

40 Bautista LE, Lopez-Jaramillo P, Vera LM, et al. Is C-reactive protein an independent risk factor for essential hypertension? J Hypertens 2001;195:857-61

41 Bautista LE, Vera LM, Arenas IA, et al. Independent association between inflammatory markers (C-reactive protein, interleukin-6, and TNF-alpha) and essential hypertension. J Hum Hypertens 2005; 19:149-54.

42 Sung K, JY S, BS K, et al. High sensitivity C-reactive protein as an independent risk factor for essential hypertension. Am J Hypertens 2003;16:429-33.

43 Sattar N, Scherbakova O, Ford I, et al. Elevated alanine aminotransferase predicts new-onset type 2 diabetes independently of classical risk factors, metabolic syndrome, and C-reactive protein in the West of Scotland Coronary Prevention Study. Diabetes 2004;53:2855-60.

44 Barzilay Jl, Abraham L, Heckbert SR, et al. The relation of markers of inflammation to the development of glucose disorders in the elderly: the Cardiovascular Health Study. Diabetes 2001;50:2384-9.

45 Pradhan AD, Manson JE, Rifai N, et al. C-reactive protein, interleukin 6, and risk of developing type 2 diabetes mellitus. JAMA 2001;286:327-34.

46 Freeman DJ, Norrie J, Caslake MJ, et al. C-reactive protein is an independent predictor of risk for the development of diabetes in the West of Scotland Coronary Prevention Study. Diabetes 2002;51:1596-600.

47 Sowers M, M J, E S, et al. C-reactive protein as a biomarker of emergent osteoarthritis. Osteoarthritis and Cartilage 2002;10:595-601.

48 Spector TD, Hart DJ, Nandra D, et al. Low-level increases in serum C-reactive protein are present in early osteoarthritis of the knee and predict progressive disease. Arthritis Rheum 1997;40:723-7.

49 Sharif $\mathrm{M}$, Elson CJ, Dieppe PA, et al. Elevated serum C-reactive protein levels in osteoarthritis. Br J Rheumatol 1997:36:140-1.

50 Streilein JW, Okamoto S, Sano Y, et al. Neural control of ocular immune privilege. Ann N Y Acad Sci 2000;917:297-306. 